\title{
Alfinev. Modelo para evaluar la alfabetización informacional en la educación superior en Cuba
}

\author{
Grizly Meneses-Placeres * \\ María Pinto-Molina **
}

Artículo recibido:

30 de mayo de 2011.

Artículo aceptado:

7 de noviembre de 2011.

\section{RESUMEN}

Se presenta el resultado final de una investigación doctoral, referida al diseño de un modelo para evaluar la Alfabetización Informacional (ALFIN) en el escenario de la educación superior. Se enuncian conceptos sobre modelo, como eje central de la propuesta. Se examinan varias experiencias sobre modelos de evaluación de otros contextos aplicables a la ALFIN a escala internacional. Finalmente se presentan los principios teóricos y metodológicos que sustentan la propuesta, se describen sus componentes y la relación entre ellos, así como la valoración emitida por expertos internacionales sobre el modelo en cuestión.

* Universidad Central "Marta Abreu” de Las Villas, Cuba. grizly@uclv.edu.cu

** Universidad de Granada, España. mpinto@ugr.es

INVESTIGACIÓN BiBLIOTECOLÓGICA, Vol. 25, Núm. 55, septiembre/diciembre, 2011, México, ISSN: 0187-358X. pp. 81-106 
Palabras clave: Evaluación; Alfabetización Informacional; Educación superior.

\section{ABSTRACT}

Alfinev. A model for information literacy assessment in cuban higher education

Grizly Meneses-Placeres and Maria Pinto-Molina

The article is the outcome of a doctoral research, related to designing a model for the evaluation of Information Literacy (IL) in higher education. Different concepts of model are presented as the core of the proposal. Various experiences of other evaluation models applied to information literacy contexts internationally are examined. Finally, the theoretical and methodological principles that support the proposal are presented, its components and the relationship between them are also described. International experts evaluate the proposal.

Keywords: Assessment; Information Literacy; Higher education.

\section{INTRODUCCIÓN}

$\mathrm{R}$ esulta significativo revisar los estudios que sobre la evaluación de ALFIN se han llevado a cabo. El propósito es establecer marcos conceptuales que les proporcionen uniformidad a investigaciones en este campo o al menos pueden considerarse como guías para futuras pesquisas.

Concebir un proceso de evaluación de la ALFIN contribuirá a la toma de decisiones de aquellos que planifiquen, desarrollen o controlen dicha actividad. En este sentido, este proceso permitirá:

1. Definir las metas, objetivos y resultados del proceso de ALFIN en la universidad.

2. Seleccionar aquellas habilidades informacionales más críticas que deba desarrollar la ALFIN en el contexto universitario, sin dejar de prepararlas todas.

3. Elegir el modo más idóneo de llevar a cabo la enseñanza de la ALFIN, 
de manera que se consiga la calidad y la transformación esperadas en la comunidad universitaria.

4. Enseñar y medir en consecuencia con esos temas y procedimientos de actuación.

Es indudable el peso tan fuerte que posee la evaluación como fenómeno, conlleva una investigación en sí misma y por tanto la conjugación de ambos elementos (ALFIN + EVALUACIÓN), sentar las pautas a nivel teórico, metodológico y práctico constituye el eje central de la presente investigación. Con este panorama se identifica como Problema de Investigación:

- ¿Cómo contribuir a la comprensión integral del proceso de evaluación de la alfabetización informacional en la Educación Superior cubana?

\section{Objetivo General}

Presentar un modelo de evaluación de la alfabetización informacional para la Educación Superior en Cuba que permita comprender el fenómeno de manera integral.

\section{Objetivos Especificos}

1. Examinar los antecedentes teóricos sobre el proceso de evaluación y evaluación de la ALFIN

2. Identificar los principios teóricos y metodológicos que sustentan el proceso de evaluación de la Alfabetización Informacional en la Educación Superior.

3. Valorar el modelo de evaluación de la alfabetización informacional en la Educación Superior cubana a partir de la consulta de expertos.

\section{Antecedentes Del estudio}

La conceptualización del proceso de evaluación es tan diversa como los autores estudiosos del tema que se encuentran en la literatura. Según Conner, Altman y Jackson, (1984) la infancia de la evaluación se sitúa en torno a los años sesenta, y su adolescencia alrededor de los ochenta, caminando en la actualidad hacia la adultez. 
Todas las definiciones son la expresión de un contexto, de una filosofía, de un modelo de pensar y entender la realidad y la práctica evaluativa. Las ciencias de la psicología y la educación han sido fundamentalmente las que han determinado su objeto y características.

Domínguez, G y Diez, (1996) establecen tres paradigmas para describir la evaluación:

1. Conductual

2. Cognitivo

3. Ecológico Contextual

Artiles Armada y Álvarez Valdivia, (1999) sostienen que la evaluación educativa consiste en "formular juicios de valor acerca de los procesos de formación de los estudiantes, para orientar las acciones educativas futuras", mientras que Rosales, (2000) indicaba que la

evaluación forma parte indisociable de la enseñanza... y actúa como un instrumento de autorregulación y perfeccionamiento dentro del proceso instructivo.

Las características de la evaluación desde esta perspectiva —ecológica contextual compartida para el diseño de ALFINEV — fueron sistematizadas por Castro, (1999):

- La subordinación de la enseñanza al aprendizaje.

- El protagonismo fluctuante del maestro y los estudiantes.

- La diversificación de los roles del maestro en la intervención, medición, coordinación y facilitación del aprendizaje de los alumnos.

- La evaluación basada en este paradigma debe estar orientada a la valoración, al análisis cualitativo de los procesos, sus estadios intermedios y los productos, con una inspiración crítica y una finalidad formativa, educativa.

La evaluación desde esta perspectiva pasa de ser analizada desde el punto de vista cuantificable y observable, a una visión totalizadora; es decir, se debe analizar tomando en consideración al sujeto y los procesos personológicos y cognitivos que lo caracterizan.

Por otra parte, estudiosos del tema como MacDonald (1971, citado en Castro 1999) se manifiestan partidarios de una evaluación holística, 
que tome en consideración todos los posibles componentes de la enseñanza: proceso, resultados, contexto... Considera que la enseñanza adquiere unas determinadas características distintas para cada situación, por lo que es necesario acercarse desde una perspectiva ecológica y contextual a la evaluación de la misma. Para este autor, finalmente; la evaluación incidirá de manera prioritaria en la búsqueda de información, para proporcionársela a quienes deben tomar decisiones.

Bajo estos preceptos teóricos, los conceptos y visiones anteriores tributan innegablemente a la aplicación de procesos de evaluación dentro de la Alfabetización Informacional, y pueden resumirse como sigue:

- La evaluación en este campo debe garantizar la sistematicidad, continuidad, flexibilidad y comunicación entre todos los componentes del proceso.

- La evaluación mantendrá una visión holística del fenómeno, para lo cual tomará en consideración los factores de índole social, cultural y ambiental existentes, que permitan el perfeccionamiento de la enseñanza y el reconocimiento del equilibrio entre los objetivos propuestos y los alcanzados.

- La evaluación se debe caracterizar por la valoración crítica de las incidencias encontradas, teniendo en cuenta no sólo el enfoque del evaluador, sino también la perspectiva del evaluado.

Por otra parte, la identificación de modelos dentro de la esfera de la alfabetización fue ampliamente analizada en la literatura científica, (Marland, 1980; Webber, 2002; Loertscher \& Woolls, 2002; Eisenberg M, 2004). De una manera explícita varios autores hacen alusión a la evaluación como otra de las etapas del propio proceso; sin embargo en la literatura revisada aparecen modelos que no son para la evaluación de ALFIN, pero que pueden ser utilizados con tal propósito. Usualmente éstos son catalogados como modelos por su amplia aplicación y son nombrados de acuerdo con sus creadores o por la técnica utilizada para la evaluación:

- Modelo de Kirkpatrick: Fue desarrollado por Donald Kirkpatrick. Tuvo una primera versión en 1959, cuyo objetivo es la evaluación de la eficacia de las sesiones de entrenamiento, de allí que sea ampliamente usada en la evaluación de ALFIN. En 1998 varios autores producen un perfeccionamiento del modelo; sin embargo la esencia se mantiene hasta el presente. El autor definió varios niveles, (Salisbury \& Ellis, 2003): 
- Nivel I: Reacción. Se conocerán los sentimientos y opiniones de los participantes, con el objetivo de saber la respuesta inmediata sobre el curso, entrenamiento o taller.

- Nivel II: Aprendizaje. Es el momento en que los estudiantes cambian sus actitudes, mejorando su conocimiento e incrementando sus habilidades como resultado de la asistencia a los programas.

- Nivel III: Comportamiento. Se relaciona con la medición de los cambios de comportamiento a largo plazo en los individuos.

- Nivel IV: Resultados. Se relacionan con el impacto o beneficio del entrenamiento a niveles organizacionales.

- Modelo de Cross \& Angelo, (Stewart, 1999): sus autores fueron Patricia Cross y Thomas Angelo. Tiene su máxima realización en el método de valoración en el aula (classroom asessment, por su significado en inglés). Consta de siete características: aprendizaje centrado en el estudiante, con la dirección del maestro en el proceso; donde exista un beneficio mutuo entre facultad/estudiante; y donde la tendencia sea tributar hacia lo formativo; se produzca en un contexto específico; se garantice la continuidad; y se caracterice por el arraigamiento de una buena práctica de la docencia. Se basa en varias asunciones, algunas de las cuales se mencionan a continuación, (Stewart, 1999: p.167):

- La calidad del aprendizaje del estudiante está directamente relacionada con la calidad de la docencia. Por lo tanto una de las formas efectivas de mejorar el aprendizaje es mejorando la docencia.

- Para mejorar su eficacia, los docentes necesitan tener claros sus metas y objetivos.

- Para mejorar el aprendizaje, los estudiantes necesitan apropiarse y focalizar tempranamente su retroalimentación, y en algunos casos necesitan aprender cómo evaluar su propio aprendizaje.

- Preguntas sistemáticas y retos intelectuales son una fuente poderosa de motivación, crecimiento y renovación para los docentes.

- Modelo de Universidad Alfabetizada en Información (UAI), (Webber y Johnston, 2006). Los autores Sheila Webber y Bill Johnston publicaron un estudio, que si bien es considerado por los autores un cuadro guía para comprender el proceso de la ALFIN en una universidad; es, además una representación de dicho proceso para medir su estadío 
—embrionario, intermedio y en buen camino hacia una UAI- en la universidad. Incluye los siguientes elementos interrelacionados:

- Gestión de la ALFIN: estrategia, recursos, política e infraestructura.

- Investigación sobre alfabetización informacional.

- Estudiantes y graduados alfabetizados en información.

- Plan de estudios alfabetizado en información: reconocimiento de la ALFIN como tema de estudio y como algo que atañe a la docencia, al aprendizaje y a la evaluación y calificación.

- Desarrollo del personal en ALFIN.

- Bibliotecarios alfabetizados en información.

El estudio de estos modelos permitió hacer una valoración de los elementos esenciales del proceso de evaluación en el contexto universitario, extrapolando varios de sus componentes al escenario del ALFIN, y permitiendo así una visión integral del proceso en el contexto cubano.

Existen otros estudios que si bien no son compartidos como modelos de evaluación de ALFIN, su propósito es estandarizar las formas de medir las habilidades informacionales en los estudiantes y la comunidad en general, y que también sirvieron de base para la propuesta final.

También las diversas posturas que existen sobre cómo evaluar la ALFIN o cuáles serían los objetos de evaluación de la ALFIN, han sido sistematizadas por Meneses, (2008) y constituyen marcos de referencia para modelar tal proceso. Otro ejemplo se ilustra en la propuesta de (Bulaong et al., 2003), quienes sólo se concentraron en la evaluación de los aprendizajes, pero que también definieron como criterios de evaluación para la ALFIN los siguientes estadíos en los estudiantes:

1. Novato

2. En desarrollo incipiente

3. En desarrollo Avanzado

4. Experto

Para cada criterio valorativo resumieron las acciones que identificarían a los aprendices en cada habilidad de información.

Resultó válida para el diseño de la propuesta del modelo la identificación de proyectos de evaluación de ALFIN. Éstos estaban mucho más enfocados a la creación de instrumentos e indicadores para efectuar la evaluación y ello permitió realizar un análisis más integral. 
Hasta el momento se reconocen proyectos encaminados a desarrollar la evaluación de la ALFIN. Su propósito es estandarizar las formas de medir las habilidades informacionales en estudiantes y la comunidad en general.

Uno de los instrumentos de mayor aplicación en campo es el proyecto SAILS, ${ }^{1}$ que fue desarrollado originalmente por un grupo de la Universidad del estado de Kent y continuado por la ACRL. El proyecto está basado en dos documentos relevantes del área de la ALFIN: Normas sobre aptitudes para el acceso y uso de la información en la enseñanza superior: estándares, indicadores de rendimiento y resultados; $y$ Objetivos para la instrucción de la ALFIN: modelo oficial para bibliotecas universitarias. Al respecto O'Connor \& et.al, (2001) plantean que el proyecto ha revelado muchas cosas sobre la evaluación, y resaltan el trabajo de sus creadores al reconocer la necesidad de incluir expertos en evaluación y de la esfera bibliotecológica, como factor crucial en el éxito del proyecto.

Dentro de los beneficios que manifiesta el mencionado proyecto se encuentran(SAILS, 2008):

1. Diseñado para darles a bibliotecarios, profesores, y administradores la información exacta sobre las habilidades de alfabetización de información de sus estudiantes.

2. Sus tests identifican las áreas dónde los estudiantes tienen mayor fortaleza en las habilidades de la alfabetización de información y aquellas que necesitan ser fortalecidas

3. Y les ofrece la oportunidad a los bibliotecarios de abordar, en conversaciones con otros colegas (profesores y autoridades) del campus universitario, la necesidad e importancia de desarrollar habilidades de información fuertes en los estudiantes universitarios.

Otro de los ejemplos es el ISKILLS creado por ETS-ICT, ${ }^{2}$ una compañía privada productora de varios instrumentos normalizados como el TOEFL. Este proyecto consiste en un examen online basado también en las Normas sobre aptitudes para el acceso y uso de la información en la enseñanza superior. Se han creado dos versiones del instrumento, una dedicada a estudiantes del primer año de la universidad y la otra más avanzada dirigida a estudiantes del tercer año en adelante. La institución encargada del diseño publicó los primeros resultados en el 2008 en conjunto con el National Literacy Policy Council. El proyecto cubre las siguientes dimensiones de la ALFIN (Sonntag, 2008).: 
1. Definición: entiende y articula el alcance de los problemas de información para facilitar la búsqueda.

2. Acceso: colecciona y recupera información de páginas webs, bases de datos, grupos de discusión etcétera.

3. Evaluación: juzga si la información resuelve el problema mediante la determinación de la autoridad, los sesgos, la obsolescencia y la relevancia de la información encontrada.

4. Gestión: organiza la información para su ayuda o para que otros la encuentren luego.

5. Integración: interpreta y representa la información, y la resume, compara y contrasta desde diversas fuentes.

6. Creación: adapta, aplica, diseña y construye información en un ambiente electrónico.

7. Comunicación: disemina información elaborada para una audiencia en particular en un efectivo formato digital.

Un proyecto que tiene muy poca divulgación en la literatura (Burkhardt, 2007) es Bay Area Community Colleges Information Competency Assessment; una nueva experiencia que se basa en 2 partes, donde se enlazan contenidos y se ofrecen respuestas cortas a interrogantes.

En Australia se inició otro proyecto de evaluación de ALFIN, el Information Skills Survey (ISS) diseñado por el Council of Australian University Librarians, se trata de un inventario de reportes personales que le pregunta a cada encuestado que describa qué hacen con la información. Está basado en las normas Australianas y de Nueva Zelanda, particularmente en los estándares del segundo al sexto,(Catts y Lau, 2008).

Una propuesta diferente de evaluación de ALFIN fue elaborada por los autores Ralph Catts y Jesús Lau, (2008). Ellos propusieron medir la ALFIN a través del diseño de indicadores de rendimiento. Revisaron tres opciones:

1. Creación de Indicadores Indirectos: abarcarían los productos de ALFIN, por ejemplo, el número de libros publicados, las patentes registradas y los volúmenes del uso de internet. Sin embargo manifestaron que estos indicadores dependían en gran medida de las condiciones económicas, y que además respondían mejor a resultados de las prácticas de ALFIN que a indicadores propios para su medición.

2. Creación de un nuevo instrumento internacional para evaluar las babilidades de información, pero que implicaba considerables costos para su desarrollo e implementación 
3. Desarrollo de un conjunto de indicadores dentro de los instrumentos internacionales ya existentes: consistían en incorporar indicadores referidos a la ALFIN dentro de ítems ya prefijados para la evaluación. Esta opción fue la más desarrollada y validada por los autores en varios instrumentos, precisamente en el Literacy Assessment and Monitoring Programme (LAMP) de la UNESCO y su Instituto de Estadística- UIS, por sus siglas en inglés-, en el Programme for International Student Assessment (PISA) y en The Demographic and Health Surveys (DHS). Todos ellos demostraron cómo era posible identificar en los parámetros de evaluación predeterminados, la inserción de ítems para comprobar la ALFIN.

\section{MÉTODOS Y TÉCNICAS}

Las clasificaciones del método científico que se utilice en una investigación estarán en dependencia del objeto de estudio y la rama del saber donde se inserte tal objeto.

A la diferente naturaleza de cada objeto de estudio, le corresponderá un método y unas técnicas de investigación que permitan captar aquello que se pretende (Rubio y Varas, 2004).

En este sentido para el desarrollo del trabajo se utilizaron los siguientes métodos de investigación científicos. Del nivel teórico:

- Histórico-Lógico: permitió la construcción de un marco teórico para conocer las particularidades e interioridades del proceso de evaluación. Y contribuyó al reconocimiento del estado actual de la problemática.

- Inductivo-Deductivo: sirvió para realizar análisis e inferencias de los datos obtenidos y permitió arribar a las regularidades y conclusiones presentadas en la investigación.

- Análisis-Síntesis: se utilizó para penetrar en la esencia del fenómeno objeto de estudio y posteriormente realizar una síntesis de la información obtenida.

En el caso del nivel empírico:

- Investigación-Acción: entendida como

la forma de búsqueda autorreflexiva, llevada a cabo por participantes en situaciones sociales (incluyendo educativas), para perfeccionar la 
lógica y la equidad de a) las propias prácticas sociales o educativas en las que se efectúan las prácticas b) comprensión de estas prácticas y c) las situaciones en las que se efectúan estas prácticas (Kemmis, 1988). Se empleó para determinar las fases incluidas en el estudio que permitieron definir los componentes finales que se incluyeron en el modelo. Fue esencialmente definitorio para discernir los objetos de evaluación de la alfabetización informacional en el contexto de la Educación Superior, para lo cual se escogieron dos especialidades de la Universidad Central "Marta Abreu" de Las Villas. ${ }^{3}$ Estas fases fueron:

1. La primera fase, con un carácter descriptivo y exploratorio, pretendió visualizar la situación preliminar del fenómeno de la ALFIN en las especialidades escogidas en el estudio. La idea fue recopilar información referida al dominio de las habilidades informacionales presentes en las comunidades de estudiantes a través del estudio en primer lugar de los planes de estudios, se realizaron sesiones con los estudiantes, con los claustros profesorales y con la biblioteca universitaria para conocer los niveles de autoevaloración con respecto a su dominio de habilidades de información y los recursos disponibles para el fomento de dicho fenómeno.

2. La segunda fase del estudio tuvo como objetivo transformar o mejorar la realidad existente. Fue la etapa decisiva dentro del estudio, pues la autora tuvo que fluctuar de investigadora a investigada. A partir del rol de docente-investigadora se incidió, desde las sesiones de las asignaturas impartidas, en la transformación a alcanzar en el dominio de habilidades de información por estudiantes, profesores y especialistas en información de la biblioteca universitaria. Además se evaluó el programa de ALFIN existente en la institución.

3. La tercera - y última - fase del estudio tuvo como propósito exponer las evidencias de la transformación o los indicios de cambios en los objetos de evaluación.

- Metodo de análisis documental. Este método se basa en la recopilación de información a partir de la consulta de fuentes de información diversas. Fundamentalmente es empleado para poder sustentar teóricamente la presente investigación. Las fuentes documentales revisadas, en su mayoría, estuvieron referidas a contrastar los conocimientos en

3 Los resultados pormenorizados de una de las especialidades escogidas para el estudio pueden encontrarse en G. Meneses Placeres, "La evaluación de habilidades de información en la licenciatura de Periodismo en la Universidad Central 'Marta Abreu' de Las Villas, Cuba”, en Documentación de las Ciencias de la Información, 2011, vol. 34. 
torno a la alfabetización informacional y las prácticas evaluativas en contextos educativos. Se resumen en los siguientes grupos:

- Documentos del área de la Educación

- Documentos del área de la Ciencias de la Información

\section{FundAMENTACIÓN TEÓRICA DEL MODELO ALFINEV}

La ALFIN puede ser considerada como una actividad docente en tanto depende de varios factores que desencadenan un proceso de aprendizaje. Es válido recordar que la ALFIN es un modo de aprender (Kuhlthau,1987 citado en Loertscher, 2002 ), por tanto existe la persona a alfabetizar y el encargado de llevar esto a cabo, a partir de la utilización de métodos, medios y procedimientos que estimularán o frenarán la acción, con unos objetivos previamente identificados. Medir la efectividad del proceso de ALFIN y realizar valoraciones al respecto, permite redireccionar, enriquecer y mejorar dichas prácticas en el escenario de la Educación Superior.

La presente investigación tiene como propósito presentar el modelo ALFINEV que contribuye a la visualización y comprensión del proceso de evaluación de la ALFIN en la Educación Superior cubana, de ahí que se imponga la consulta y consideración general de modelo.

Según el Diccionario Filosófico un modelo se define como:

la reproducción del objeto que se investiga en otro análogo que se construye y que recibe el nombre de modelo (Diccionario Filosófico, 1973).

Desde los estudios vinculados a la lógica y al análisis del pensamiento encontramos que el modelo se define

como un objeto artificialmente creado en forma de estructura física, esquema, fórmula de signos, etc.; que se asemeja al objeto original y refleja sus principales características, relaciones, estructura, propiedades (Guetmanova, 1991).

Por su parte el Diccionario Cervantes de la Lengua explica al modelo como:

1/ Objeto que se reproduce imitándolo. 2/ Representación en pequeña escala. 3 / Persona, animal u objeto que reproduce el pintor o escultor. 4/ Persona o cosa digna de ser imitada. 5/ Tipo industrial protegido por una patente: modelo registrado. 6/ Mujer que en las casas de modas exhibe los nuevos modelos de costura. 7/ Adj. Perfecto en su género: un alumno modelo, (Diccionario Cervantes, 1998). 
Mientras que desde el punto de vista epistemológico el modelo es

toda construcción teórica que sirve para interpretar o representar la realidad o una parte de la realidad (...) modelo es una manera de interpretar o explicar la teoría o parte de la teoría científica, acercando lo abstracto a lo concreto o a la teoría a la realidad, (Diccionario de Filosofía, 2002).

Para De Armas Ramírez, (2003) modelo es

una construcción general dirigida a la representación del funcionamiento de un objeto a partir de una comprensión teórica distinta a las existentes.

Con el estudio de la diversidad de procedencia de los conceptos referidos a modelo, se alude a que todos ellos sistematizan la expresión de que es una representación — teórica o gráfica- de un determinado fenómeno u objeto. La idea es reflejar de una forma abstracta, y a su vez precisa, los elementos fundamentales que caracterizan tal fenómeno. Lo importante es que sea capaz de entenderse en la comunidad científica a la cual está dirigida. Por tanto, en este estudio se concuerda con la definición donde se concibe al modelo como

la representación de aquellas características esenciales del objeto que se investiga, que cumple una función heurística, ya que permite descubrir y estudiar nuevas relaciones y cualidades de ese objeto de estudio con vistas a la transformación de la realidad (Del Valle Lima, 2007).

La definición asumida se fundamenta a partir de que la propuesta ofrece relaciones diferentes a las encontradas en la comunidad científica internacional sobre la evaluación de la ALFIN en el contexto de la Educación Superior. Permite entender este proceso desde una arista más integradora del fenómeno en sí y desde cada uno de sus componentes por separado. Se analizan a profundidad tales relaciones y se contribuye al conocimiento más directo del proceso de evaluación y que favorece su transformación desde la praxis universitaria. La propuesta organiza los modos de comprensión, análisis y concepción de sus componentes, así como su inter e intra dependencia a través de las características esenciales.

El modelo se fundamenta en la Pedagogía y la Psicología como ciencias y en las Ciencias de la Información. En la concepción del modelo se asume desde el punto de vista Psicopedagógico que:

1. Los objetos de evaluación en el contexto educativo son en primera instancia el aprendizaje de los alumnos, además del profesor, los recursos 
didácticos y la interacción entre todos.

2. La evaluación de procesos docentes y lógicamente la evaluación del aprendizaje dependen de (Enciclopedia General de Educación, 2000):

- Se aprende mejor cuando se está dispuesto a aprender.

- Cuanto más se pone en práctica lo aprendido, mejor se entiende.

- Se recuerda mejor lo aprendido cuando ha sido útil, beneficioso o satisfactorio.

- Aprender algo nuevo es más fácil cuando está basado en lo que ya se sabe.

- Se aprende hacienda (learning by doing de J Dewey).

Desde el punto de vista Informacional:

1. Se debe entender la información desde sus disímiles expresiones, portadores y escenarios. Este postulado orientó la propuesta, pues permitió entender el fenómeno de la alfabetización y su implicación como proceso de formación permanente a lo largo de la vida.

2. La información se relaciona estrechamente con el aprendizaje como resultado de continuos procesos de construcción socio-cultural, donde la alfabetización informacional constituye uno de los procesos que subyacen en tal construcción.

3. Las Ciencias de la Información son consideradas como una ciencia interdisciplinar. Por ello la propuesta, diseñada para enriquecer el arsenal teórico del que la conforma, se sustenta en la conjugación de principios provenientes de otras ciencias sociales.

4. Desde esta ciencia se han determinado como elementos necesarios para estar en presencia de la ALFIN, el dominio de las siguientes habilidades de información:

- determinar el alcance de la información requerida,

- acceder a ella con eficacia y eficiencia,

- evaluar de forma crítica la información y sus fuentes,

- incorporar la información seleccionada a su propia base de conocimientos,

- utilizar la información de manera eficaz para acometer tareas específicas y

- comprender la problemática económica, legal y social que rodea al uso de la información, accediendo a ella y utilizándola de forma ética y legal. 
A partir de esta interpretación se asumen en el modelo, cuya función esencial es la de una evaluación formativa, los siguientes principios:

1. Propiciar ambientes de aprendizajes que ofrezcan a los estudiantes la oportunidad de desarrollar habilidades informacionales.

2. Valorar al proceso de alfabetización Informacional desde una función formativa y por tanto sistémica que depende de todos los componentes que la integran para cumplimentar su meta.

3. El objeto de evaluación de la alfabetización informacional no se concentra solamente en los niveles de aprendizajes logrados por los estudiantes.

4. Hay que considerar la conjugación de instrumentos novedosos que garanticen una evaluación auténtica del proceso de ALFIN.

5. La propuesta toma en consideración los conocimientos y habilidades, informacionales necesarias en el nivel superior del colectivo de profesionales encargados del proceso.

6. Se respeta la visión holística de cada objeto de evaluación de ALFIN considerando las funciones diagnóstica y formativa del proceso valorativo

Las exigencias básicas de la concepción que dan sustento a la propuesta son las siguientes:

- Comprensión y disposición de los profesionales encargados del proceso de involucrarse en las actividades de ALFIN.

- Formalización de espacios que potencien el desarrollo de la ALFIN desde lo curricular, y que faciliten continuamente el progreso del aprendizaje de los estudiantes

- Implicación consciente de los estudiantes en el proceso de ALFIN.

\section{OBJETIVO Y CARACTERÍSTICAS DEL MODELO ALFINEV}

Nombre: ALFINEV. Modelo para evaluar la ALFIN en la Educación Superior en Cuba.

El modelo que se presenta tiene como objetivo general: representar el proceso de evaluación de la ALFIN en la Educación Superior de manera que permita la comprensión integral del fenómeno.

Dentro de las características generales del modelo se encuentran: 
1. Integra la visión de biblioteca-facultad como binomio esencial para la consecución del proceso efectivo de ALFIN.

2. Considera el tratamiento de los profesionales encargados del proceso a un mismo nivel en lo que a la ALFIN se refiere.

3. Amplía la visión de los recursos didácticos, indispensables como objeto de evaluación en el contexto educativo, pues no ubica solamente el análisis de los componentes del proceso de enseñanza aprendizaje, sino que extiende su concepción a programas, planes de estudios etcétera.

4. Posee un carácter sistémico, pues establece la inter e intradependencia de todos sus componentes para lograr la efectividad del proceso de evaluación de ALFIN. No puede llevarse a cabo este proceso de valoración sin la confluencia de alguno de los componentes preestablecidos.

5. Tiene un carácter cíclico, pues se concibe a la evaluación como proceso que no termina con una calificación de habilidades de información o el otorgamiento de un certificado acreditativo, ya sea a profesores o estudiantes, sino con alcanzar niveles superiores en ese propio desarrollo de las habilidades informacionales que desencadenen nuevas necesidades de información y mayor profundidad en la demostración de tales habilidades.

A partir de estas características, estos elementos fueron considerados como necesarios para concebir un funcionamiento adecuado del modelo propuesto. Éstos devinieron de las dimensiones e indicadores encontrados en las regularidades del estudio y pertenecen a los objetos de evaluación de la ALFIN identificados:

- El diagnóstico de los niveles de destreza en las habilidades de información de los estudiantes universitarios a través de la aplicación de cuestionarios y la realización de grupos focales como bases del proceso evaluativo.

- La evaluación diagnóstica inicial se debe continuar con una evaluación continua, lo que implica determinar las potencialidades desde el plano curricular y extracurricular.

- El diseño de actividades docentes y evaluativas que incorporen y midan las habilidades de información en el ejercicio de su futura profesión.

- La preparación tanto pedagógica e informacional del colectivo inmerso en el proceso de formación de ALFIN.

- La efectividad de un programa de ALFIN rectorado por la institución de información universitaria. 
- El examen de los antecedentes de formación de habilidades de información tanto en los estudiantes como en los profesionales.

- La revisión de políticas universitarias en cuanto al uso de la información y su nivel de aplicación.

- La generación de materiales didácticos desde la esfera extracurricular con vistas a consolidar las habilidades de información.

- El equilibrio entre los conocimientos, destrezas y actitudes en cuanto al manejo y uso de la información.

\section{COMPONENTES DEL MODELO ALFINEV}

A partir de las disposiciones teóricas analizadas se determinaron los siguientes componentes que reflejan el proceso de evaluación de la ALFIN, (Fig. 1):

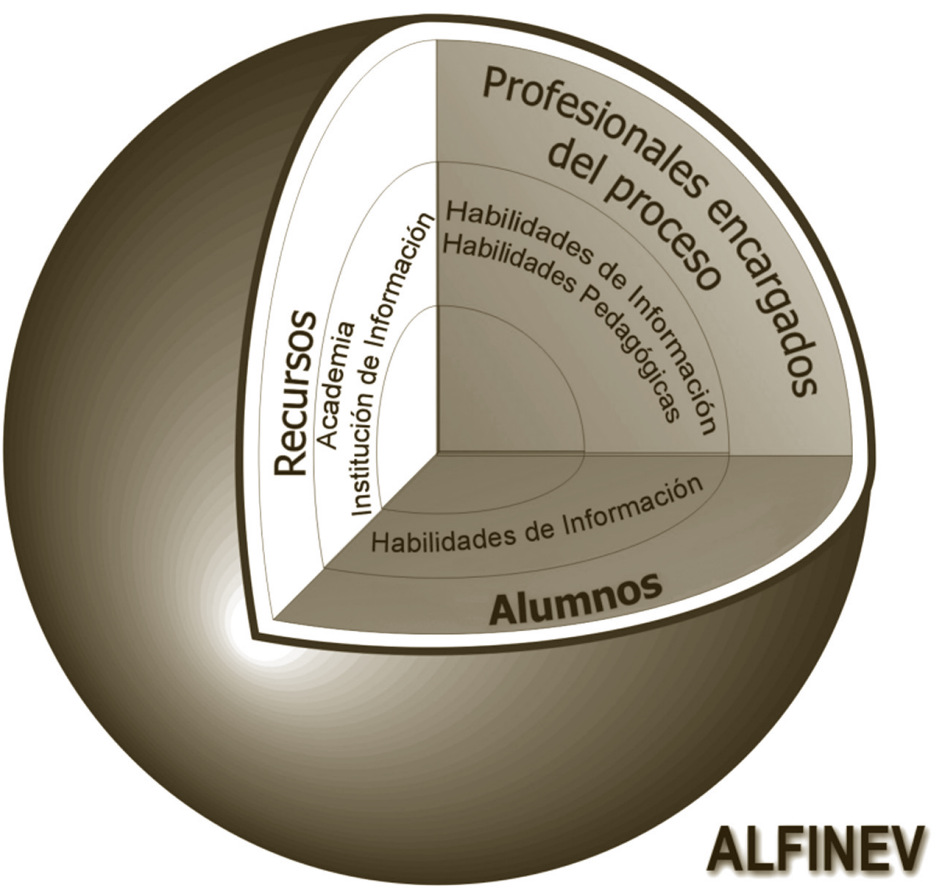

Fig.1. ALFINEV: modelo para evaluar la ALFIN en la universidad cubana 


\section{Profesionales encargados del proceso.}

Esta categoría hace referencia a dos aspectos fundamentales: el personal docente, en este caso al profesor universitario quien debe ser el artífice de la promoción de la ALFIN en sus estudiantes, y el profesional de la información, es decir, la figura del especialista de la biblioteca universitaria.

En la literatura científica se ha aludido, en disímiles estudios, a la relación estrecha que debe existir entre el docente y el bibliotecario para el desempeño exitoso de procesos de ALFIN; y que al final repercutan directamente en el aprendizaje de los alumnos: uno desde el propio curriculum, el otro apoyando, tanto desde lo curricular como desde lo extracurricular. Los modelos revisados, en algunos casos, mencionan la presencia del bibliotecario de manera explícita, (ACRL, 2005; Colborn y Cordell, 1998; Webber y Johnston, 2006), en otros se señala a la institución de información, y en terceros no se refieren al profesorado — (Gratch Lindauer, 2004)—, visiones estas que continúan lacerando la propia relación. Sin embargo, se concibe como novedoso en la propuesta ver todo esto desde una misma arista. Se trata de entender al docente y al bibliotecario en un mismo nivel de implicación para el desarrollo del proceso de ALFIN en la universidad.

Independientemente de las intenciones de colaboración, las acciones de ALFIN pueden no lograr su objetivo si, por una parte el profesor no posee la habilidades informacionales necesarias para formarla o consolidarlas en sus estudiantes dentro del proceso docente, o por la otra si el bibliotecario no utiliza técnicas participativas y, en el mejor de los casos, cuando se utilicen estrategias didácticas en función de lograr transmitir — tanto a estudiantes como a profesores- sus saberes en torno a la búsqueda, localización, acceso, evaluación y uso de la información de manera efectiva.

En este sentido, la preparación pedagógica e informacional de tal binomio resulta un indicador significativo en la consecución de un proceso valorativo sobre la ALFIN en la Enseñanza Superior.

Esta relación se acentúa más, pues al ubicarlos a un mismo nivel de incidencia, las debilidades de unos pueden constituir fortalezas en el otro y así generarse procesos de perfeccionamiento dentro del propio componente. Tal es el caso de la preparación que pueden ofrecer los bibliotecarios en alguna habilidad de información que contribuya al progreso de los profesores.

\section{Recursos}

Por esta categoría se entiende todos los medios, los métodos y procedimientos empleados en la Enseñanza Superior que tributen a la ALFIN. De este modo, 
se identificaron varios elementos, entre los cuales se vuelven a conjugar biblioteca-facultad.

En este sentido se prevee desde los planes de estudios de las carreras, las clases y su preparación por los docentes, hasta el programa de ALFIN diseñado por la biblioteca universitaria, la inclusión de las sesiones y cursos impartidos en ella, el diseño de tutoriales en línea, los recorridos por la instalación etcétera.

Estos recursos también ofrecen la visión sistémica e intradependiente, pues en el propio programa de ALFIN existente en la biblioteca se insertan contenidos en asignaturas del plan de estudios universitario, o desde la academia se pueden proponer acciones desde el plano curricular, metodológico donde se aprovechen los espacios de la misma en el desarrollo de la ALFIN tanto los estudiantes como los profesores.

\section{Alumnos (aprendizaje de habilidades de información)}

En esta categoría es donde se concentran los resultados propios del proceso de evaluación de ALFIN. Es aquí donde se constata el dominio y progreso de las habilidades informacionales de los estudiantes. En este sentido, las normas establecidas por la ALA (2000) para estudiantes de Educación Superior en cuanto al acceso y uso de la información de forma efectiva, constituyeron el marco de apoyo para sustentar el estudio, al englobar desde su concepción misma la triada conocimiento-habilidades-aptitudes para el manejo de información.

Se consideró el nivel de conocimientos que poseen los estudiantes sobre las habilidades de información, que les permitan formularse juicios de valor con respecto a ellas para su desarrollo profesional y de autoevaluación sobre el dominio de tales habilidades informacionales, además de la compresión desde una arista actitudinal del empleo de estas habilidades. Para ello se revisaron los niveles de independencia y criticidad de los estudiantes para determinar las formas o espacios donde perfeccionar y revertir la situación desfavorable o no encontrada de dichas habilidades.

\section{Interacción entre los componentes}

La representación gráfica del fenómeno refleja la relación estrecha entre cada uno de los componentes. La idea del círculo representa la concepción misma de la evaluación, es ver los componentes como entes dependientes para el avance de la ALFIN en la universidad. Ninguno por encima de otro, sino como un todo. 
ALFINEV muestra la simulación de anillos como corteza de un árbol en cada uno de sus componentes. Éstos representan un proceso significativo al que la ACRL en su propuesta hace referencia: transferibilidad. Es decir, estudiar la incidencia de actividades en enseñanzas o asignaturas precedentes para analizar el adecuado proceso de ALFIN en el presente, pero ALFINEV no sólo ve este ciclo en el aprendizaje de los alumnos, sino también en el resto de los componentes. En el caso de los profesionales sería considerar en espiral su preparación para enfrentarse a la ALFIN — tiempo dedicado a la actividad, procedencia, dominio de habilidades y forma de su obtención o consolidación-. Por otra parte los recursos, deben ser examinados también desde esa perspectiva.

La interdependencia entre los componentes también puede valorarse a partir de las siguientes premisas:

- La calidad de los recursos depende de la calidad y preparación de los profesionales.

- Los estudiantes podrán concientizar más su rol en la consolidación de las habilidades de información si los profesores convierten a éstas en una práctica cotidiana en sus aulas.

- La generación de materiales didácticos, sesiones y talleres, en definitiva el diseño del programa de ALFIN en la institución de información podrá ser enriquecido y mejorar si parte de una evaluación constante de sus usuarios finales, los estudiantes y profesores.

\section{VALORACIÓN DEL MODELO ALFINEV POR CRITERIOS DE EXPERTOS}

El modelo ALFINEV propuesto se sometió a la valoración de los criterios de expertos. Se asumió la metodología propuesta por la Metodología de Crespo (2007), donde se les asigna un valor a los expertos a partir de sus fuentes de argumentación. Para la selección de los expertos se procedió de la siguiente forma:

- Preselección de los posibles expertos sobre la base del conocimiento que posee la investigadora.

- Determinación de los expertos según la competencia, refrendada en las respuestas al cuestionario sobre su autovaloración del fenómeno analizado.

- Determinación del coeficiente K, a partir de la opinión del encuestado de acuerdo con el nivel de conocimiento sobre el problema investigado y a través de las fuentes que le permiten esa fundamentación. 
- Selección de los expertos para la segunda fase del proceso. Se contó con 11 expertos en total. La Tabla 1 muestra su distribución general.

- El grupo de expertos respondió un segundo cuestionario donde se valoraron los principios que sustentan el modelo ALFINEV, en una escala de Muy importante, Bastante importante, Importante, Poco importante, No importante.

- Finalmente se valoró la funcionalidad del modelo en una escala de 5 (Imprescindible para lograr la funcionalidad del modelo); 4(muy útil para lograr la funcionalidad del modelo); 3 (útil para lograr la funcionalidad del modelo); 2 (quizás podría servir para lograr la funcionalidad del modelo); 1- no aporta nada a la funcionalidad del modelo.

Tabla 1: Caracterización de los expertos escogidos

\begin{tabular}{|c|l|c|}
\hline Expertos & \multicolumn{1}{|c|}{ Centro de procedencia } & Años de experiencia en Alfin \\
\hline 1 & Universidad de La Habana, CUBA & 8 \\
\hline 2 & Universidad de La Habana, CUBA & 7 \\
\hline 3 & Universidad Agraria de La Habana, CUBA & 5 \\
\hline 4 & Instituto Federal, MÉXICO & 2 \\
\hline 5 & Dirección Informatización MES, CUBA & 5 \\
\hline 6 & Escuela Interamericana de Bibliotecología, COLOMBIA & 5 \\
\hline 7 & El Colegio de México, MÉXICO & 8 \\
\hline 8 & Universidad Adolfo Ibáñez, CHILE & 5 \\
\hline 9 & Universidad de Granada, ESPAÑA & 12 \\
\hline 10 & Universidad de Salamanca, ESPAÑA & 10 \\
\hline 11 & Universidad Autónoma de Ciudad Juárez, MÉXICO & 15 \\
\hline
\end{tabular}

Los criterios expresados por los expertos alrededor de los principios generales que sirvieron de base al diseño del modelo y su funcionalidad, se enfocaron en:

- El 100\% de los expertos considera como muy importante propiciar ambientes de aprendizajes que les ofrezcan a los estudiantes la oportunidad de desarrollar habilidades y actitudes en el manejo y uso de la información.

- Un experto considera que no es importante concentrar la evaluación de la ALFIN en otros objetos diferentes al aprendizaje de los estudiantes; sin embargo el 90\% lo cataloga en las categorías de muy importante y bastante importante.

- Entender el rol de la Educación Superior en la sociedad actual, así como las particularidades de las diversas especialidades, condicionan 
un uso eficiente de la información, algo considerado por el $72.2 \%$ de los expertos como muy importante, mientras que el 18.1\% afirma que es bastante importante.

- El 82\% de los expertos valoró como muy importante tener una visión holística de cada objeto de evaluación de ALFIN incluido en la propuesta, mientras el 18\% ubica este aspecto como bastante importante.

- El 82\% considera imprescindible para el funcionamiento efectivo del modelo el diagnóstico de los niveles de destreza en las habilidades de información de los estudiantes universitarios a través de la aplicación de cuestionarios y la realización de grupos focales como bases del proceso evaluativo, y el $9 \%$ cree que es muy útil.

- Con respecto a la preparación pedagógica e informacional del colectivo inmerso en el proceso de formación de ALFIN, el 100\% de los expertos piensa que es imprescindible o muy útil para el funcionamiento del modelo.

- E1 81\% de los expertos asignaron la categoría de imprescindible o muy útil para el funcionamiento del modelo el equilibrio entre los conocimientos, destrezas y actitudes sobre el manejo y uso de la información. Sin embargo el restante $18 \%$ lo valora de útil.

- La efectividad de un programa de ALFIN rectorado por la institución de información universitaria fue un indicador para medir la funcionalidad del modelo. El 81\% de los criterios lo evalúan como imprescindible o muy útil para su consideración. Un 9\% lo valora de últil y un solo experto entiende que quizás pueda servir.

- ALFINEV establece como premisa, y a su vez determina su funcionalidad, el examen de los antecedentes de formación de habilidades de información tanto en los estudiantes como en los profesionales. Este aspecto fue valorado por los expertos, quienes le otorgaron en un $63 \%$ la categoría de imprescindible o muy útil, sin embargo el $28 \%$ afirma que es últil.

\section{Conclusiones}

1. La evaluación ha sido descrita a través de paradigmas fundamentales: conductual, congnitivo y ecológico contextual, siendo este último la visión más compartida por la comunidad cientifica actual, en tanto toma en consideración los factores de índole social, cultural y ambiental existentes que permiten el perfeccionamiento de la enseñanza.

2. La investigación intervino en cada objeto de evaluación de ALFIN 
identificado, y logró transformaciones para su mejora. En este sentido la comunidad de estudiantes, en el escenario de las asignaturas, demostró su avance en los niveles de destrezas de las habilidades de información, a partir de su participación en exámenes de premios, calidad y presentación de la información en los informes finales. De igual forma los docentes y especialistas recibieron acciones de entrenamiento y superación que contribuyeron al progreso de sus habilidades informacionales, y la biblioteca universitaria reconoció la importancia de rediseñar su programa de ALFIN para favorecer su inserción en la enseñanza universitaria.

3. Los principios generales que sustentan el modelo propuesto están referidos a: la creación de ambientes de aprendizajes que potencian el desarrollo de habilidades informacionales; la concepción sistémica y la visión holística de todos los componentes; que el objeto de evaluación de la alfabetización informacional no se concentre solamente en los niveles de aprendizaje logrados por los estudiantes; que se considere la conjugación de instrumentos novedosos que garanticen una evaluación auténtica del proceso de ALFIN; y que se tomen en consideración los conocimientos y habilidades informacionales del colectivo de profesionales encargados del proceso desde una función diagnóstica de la evaluación.

4. Los componentes del modelo ALFINEV propuesto son los profesionales y sus habilidades informacionales y pedagógicas, los recursos disponibles que favorecen la ALFIN elaborados desde la academia o desde la institución de información, y las habilidades de información de los estudiantes.

5. El 100\% de los expertos valoró los principios generales donde se sustenta la propuesta entre las categorías de Muy Importante y Bastante Importante. De la misma manera evaluaron las características fundamentales que garantizan su funcionalidad en los rangos de Imprescindible y Muy útil para lograr su efectividad.

\section{Referencias Bibliográficas}

ACRL, "Agenda para la investigación en instrucción bibliográfica y alfabetización informacional (alfin)", en Anales de la Documentación, 2005;8:275-83.

ALA, Normas sobre aptitudes para el acceso y uso de la información en la enseñanza superior, 2000 [updated 2000; cited 200410 febrero]; available from: http://www.ala.org/ala/acrl/acrlstandards/informationliteracycompetencystandards.htm. 
Artiles Armada, K. y Alvarez Valdivia, I. M., La evaluación en la universidad: estudio preeliminar, Centro de Estudio de Educación, Universidad Central de Las Villas Santa Clara; Villa Clara, Cuba, 1999.

Burkhardt, J. M., "Assessing Library Skills:A First Step to Information Literacy", en Portal: Libraries and the Academy, 2007, vol. 7, núm. 1, pp. 25-49.

Bulaong, G.; Hoch, H., et al, "Criterios para la competencia o alfabetismo informacional en la enseñanza superior", en Developing research and communication skills: guidelines for information literacy in the curriculum, Philadelphia: Middle States Commission on Higher Education, 2003.

Castro, O., Evaluación Integral del Paradigma a la Práctica, La Habana: Pueblo y Educación, 1999.

Catts, R. y Lau, J., Towards information literacy indicators, París: UNESCO, 2008, $44 \mathrm{p}$.

Colborn NW, Cordell RM, "Moving from Subjective to Objective Assessments of Your Instruction Program", en Reference Services Review, 1998;26(3-4):125-37.

Colectivo de autores, Diccionario Cervantes, Madrid, 1998, Página 978.

Colectivo de autores, Diccionario Filosófico, Ediciones Revolucionarias, Habana, 1973.

Colectivo de autores, Diccionario de Filosofía en CD ROM, Editorial Herder, 2002, España.

Colborn, N. W. y Cordell, R. M., "Moving from Subjective to Objective Assessments of Your Instruction Program", en Reference Services Review, 1998, vol. 26, núm. 3-4, pp. 125-137.

Crespo, T., Respuestas a 16 preguntas sobre el empleo de expertos en la investigación pedagógica, Perú: San Marcos, 2007.

De armas Ramírez, N., "Caracterización y diseño de los resultados científicos como aporte de la investigación educativa”, en Pedagogía 2003, Palacio de Convenciones, Ciudad de La Habana, 2003.

Del Valle Lima, A., Metamodelos de la investigación pedagógica, Ciudad de La habana: Instituto Central de Ciencias Pedagógicas.Ministerio de Educación, 2007.

Domínguez, G. y Diez, G. "La Evaluación del Funcionamiento de un Centro a través del análisis de su Cultura Organizativa como Instrumento para la Mejora y la Innovación”, en Domínguez, G. y Mesona, J. (editor), Manual de Organización de Instituciones Educativas, Madrid: Escuela Española, 1999.

Eisenberg M., Information Literacy: Ensuring effective use of information. 2004 [updated 2004; cited 200427 enero]; Available from: http://www.ischool.washington.edu/mbe/presentations/ Info\%20Literacy-University\%20of\%20Puerto\%20Rico\%2022004.ppt 
Enciclopedia General de Educación, Barcelona: Océano, 2000, vol. Tomo II.

Gratch Lindauer, B., "Definición y medida del impacto de las bibliotecas universitarias sobre los resultados globales de la institución”, Boletín de la Asociación Andaluza de Bibliotecarios, 2004, núm. 59.

Guetmanova, A \& et. al, Lógica: En forma simple sobre lo complejo (Diccionario), Ed. Progreso, Moscú, 1991, p. 234, tomado de Ordaz Lorenzo, R. La modelación como método científico general del conocimiento y sus potencialidades en el campo de la educación, ISPEJV, 2003, Material en soporte magnético.

Kemmis, S., El curriculum. Más allá de la teoría de la reprodución, Madrid: Morata, citado en Rodríguez Gomez, G., Gil Flores, J. \& García Jimenez, E. (2004), Metodología de la investigación cualitativa, La Habana, Félix Varela, 1988.

Loertscher D, Woolls B., Information literacy: a review of the research. A guide for practitioners and researchers, California: Hi Willow Research and publishing; 2002.

Marland M., Information Skills in the secondary curriculum: the recommendations of a working group sponsored by the British Library and the Schools Council, London: Methuen Educational for the School Council; 1981

Meneses Placeres, G. "Aproximaciones teóricas a la evaluación de la alfabetización informacional en la Educación Superior”, en ACIMED, 2008, vol. 18, núm. 1.

O'Connor LG, Radcliff CJ, Gedeon JA. Applying Systems Design and Item Response Theory to the Problem of Measuring Information Literacy Skills, College \& Research Libraries, 2002(6).

Portmann CA, Jilius Roush A., "Assessing the effect of library instruction", en The Journal of Academic Librarianship, 2004;30(6):461-5.

Rosales C., Evaluar es reflexionar sobre la enseñanza, Madrid: Narcea; 2000.

Rubio, M. J. y Varas, J., El análisis de la realidad en la intervención social. Métodos y técnicas de investigación, 3ra ed. Madrid: Editorial CSS, 2004.

SAILS Proyect, Sails: Standarized Assessment Of Information Literacy Skills, (Brouchure), Kent University, 2008.

Salisbury, F. y Ellis, J., "On line face to face: evaluating methods for teaching information literacy skills to undergrate arts students", en Library Review, 2003, vol. 52, núm. 5, pp. 209-217.

Stewart SL., "Assessement for library instruction: The Cross/Angel Model!, en Research Strategies, 1999;16(3):165-74.

Sonntag, G., "We have evidence, they are learning: using multiple assessments to measure student information literacy learning outcomes", en World Library and Information Congress: 74Th Ifla General Conference and Council, Quebec. 10-14 agosto 2008. 
Webber S., Information literacy: definitions and models 2002, [updated 2002; cited 200412 febrero]; available from: http://dis. shef.ac.uk/literacy/definitions.htm.

Webber, S. y Johnston, B., "Hacia una universidad alfabetizada en información según Sheila Webber y Bill Johnston”, en Boletín de la Asociación Andaluza de Bibliotecarios, 2006, núm. 84-85, pp. $47-$ 52. 